\title{
Interactive cartographic visualization of real-time satellite data and their orbits
}

\author{
Shlesha Acharya \\ Technical University of Munich, shlesha.ach@gmail.com
}

Keywords: interactive real-time maps, real-time data, satellite orbit visualization, user-centered design

\begin{abstract}
:
The paradigm shift from the static paper map to an interactive web map and gradually into regularly updated real-time maps require the involvement of various cartographic techniques. The excessive use of web maps is the result of easy access to the internet and Geographic Information Systems. The use of dynamic web maps for visualizing real-time data is increasing in recent years for both expert and general map users (Tolochko, 2016). Furthermore, various fields such as criminology, natural hazards, climate change, social cause, aerospace, cartographic research are adopting real-time maps. The adaptation of interactive real-time maps in various fields has challenged cartographic researchers to find proper ways of visualization in scientific, technological, and aesthetic realms. This results in the need for effective cartographic techniques, guidelines, and interactive elements in preparing web-based interactive real-time maps.

For this study, various operational and junk satellites are taken to develop a real-time dataset that is represented in an interactive web-based map. These complex time-dependent data are suitable for investigating novel cartographic visualization approaches because they challenge the existing practices. This research aims to optimize the cartographic visualization of real-time satellites and their orbits. Although numerous web-based applications are in practice for this purpose, the cartographic aspects of visualization, comprehensive menu navigation, and user-friendliness seem to have been neglected. Further, there is a lack of proper guidelines for visualizing satellite orbits in the case of the interactive real-time map. Hence, this research presents the minimum criteria and interactions required for satellite orbit visualizations.
\end{abstract}

Additionally, the cartographic interactions and user interface design required for the preparation of an interactive map have been explored. For this purpose, an interactive real-time web map has been developed taking the user-centered design workflow (Needs Assessment - Prototyping - Implementation - Deployment - Maintenance), data management, and interactions (Pan and Zoom, Retrieve, Search and Filter, Overlay) (Tolochko, 2016). The two-line element (TLE) data is used to retrieve the position and orbit of the satellite. TLE datasets are one of the greatest sources for tracking data for satellites in Earth's orbit. Any object greater than 10 centimeters present in the space can have its respective TLE datasets (Kardol, 2018).

The efficiency, effectiveness, and satisfaction of a map are checked with the help of a user study consisting of pre-test and post-test questionnaires. The pre-test questionnaire will help to understand the user's general background. The posttest questionnaires will help to understand the experience of the user while using the application. The users for this study have been divided into two groups: (1) the users having an experience of using interactive maps frequently, and (2) the users having experience of using interactive maps only for a specific reason.

An interactive High-Fidelity prototype of a web map was built with the browser-based user interface design application, FIGMA. It allows various interactive tools for the user study. The first phase of the user test is done with the help of this prototype and once revised and approved, the prototype is implemented using HTML, CSS, JavaScript to generate a web-based map. The second phase of the user test is done with the above-mentioned implemented prototype to perform the final analysis to obtain the research objectives.

The research questions (RQ) for the above-mentioned objectives are the following: RQ1. Are 2D or 3D maps more effective for displaying satellite orbits on an interactive web-based map? RQ2. What color or other graphic variable choices (shape, transparency, texture, line-type, and line-weight) are most effective at illustrating the orbital paths of satellites? RQ3. How can two or more user-selected satellites be visualized together for a comparison? To find the answer to the aforementioned RQ1 and RQ3, the interface of the prototype was designed which is presented in Figure 1 and Figure 2. The preview of some interactions to customize the interface by the map users are represented in Figure 3 and Figure 4. The findings to these questions will help to determine the optimized design of a real-time interactive satellite map. 


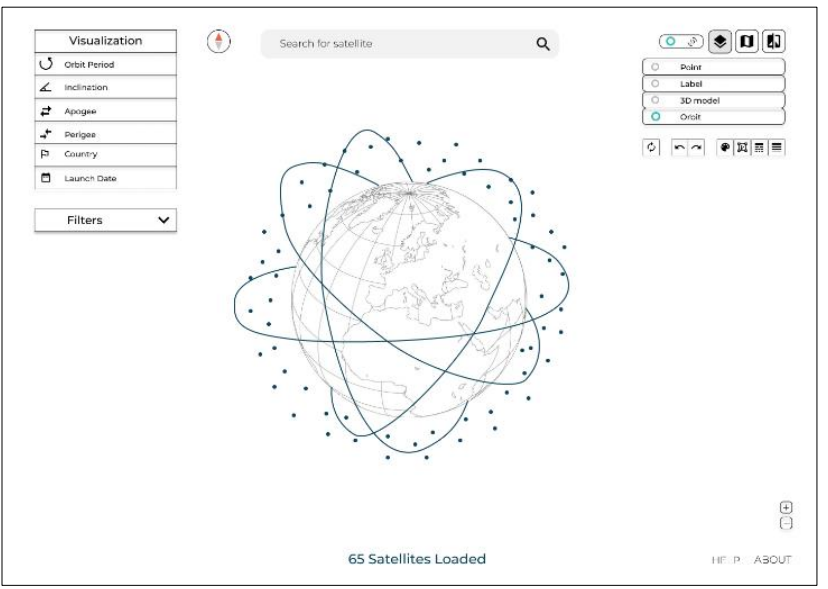

Figure 1. The preview of the visualization of satellites and their orbits with a $3 \mathrm{D}$ virtual globe.

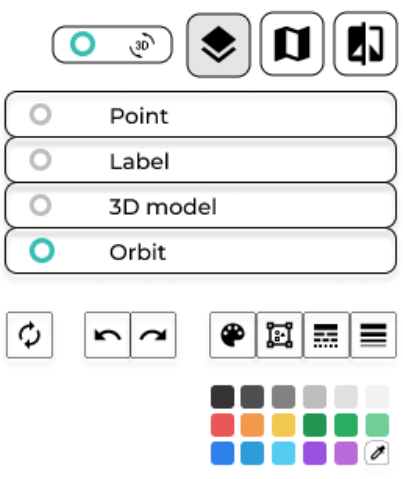

Figure 3. The preview of the customizable color variable for the users to customize the interface.

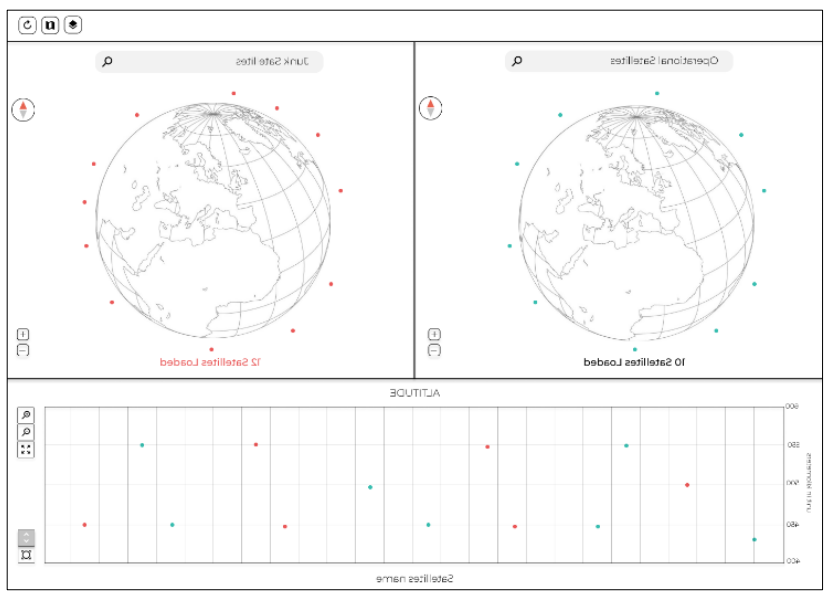

Figure 2. The preview of the interface to visualize the comparison of two or more user-selected satellites.

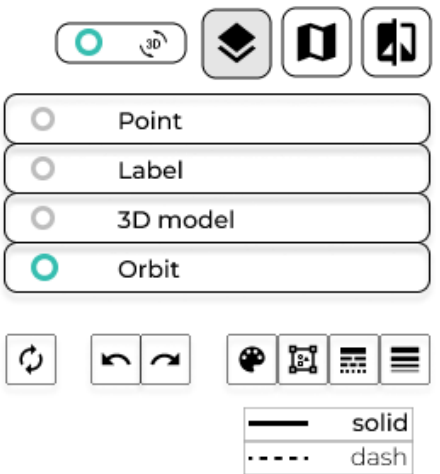

Figure 4. The preview of the line-type variable for the users to customize the interface.

In conclusion, this research explores the required guidelines and interaction for the preparation of interactive web-based applications using real-time satellite data. The findings are not only useful for the visualization of satellite data but also for various kinds of real-time data. The web-based application so designed will contribute to the visualization of planet constellations, and space debris. It also plays an important role in detecting the collision of satellites since the application allows the comparison of multiple satellites. This study will be a contribution to the field of cartography and serve as guidance to future researchers in the visualization of satellite data with an interactive real-time map.

\section{References}

Kardol, S. (2018). An interactive, web-based, near-Earth orbit visualization tool. https://repository.tudelft.nl/islandora/object/uuid\%3A9be69727-0493-4595-92d8-c7598d8fb876

Tolochko, R. (2016). Contemporary Professional Practices in Interactive Web Map Design. 103. 